\title{
Erratum
}

\section{The KPSS Test with Outliers}

\section{JESÚS OTERO ${ }^{1}$ and JEREMY SMITH ${ }^{2}$}

${ }^{1}$ Facultad de Economía, Universidad del Rosario, Bogota, Colombia

${ }^{2}$ Department of Economics, University of Warwick, Coventry, CV4 7AL, UK;

E-mail: jeremy.smith@warwick.ac.uk

Accepted 2 November 2005

Unfortunately the page numbers online for this article are incorrect. The correct page numbers should be: 241-249. 